\title{
Impacto do uso da terra na eficiência do sequestro de carbono no Sul da Amazônia
}

\author{
Verônica Satomi KAZAMA ${ }^{1^{*}}$, Ana Paula Dalla $\mathrm{CORTE}^{2}$, Franciel Eduardo REX', \\ Aline Bernarda DEBASTIANI ${ }^{1}$, Carlos Roberto SANQUETTA ${ }^{2}$ \\ ${ }^{1}$ Programa de Pós-Graduação em Engenharia Florestal, Universidade Federal do Paraná, Curitiba, PR, Brasil. \\ ${ }^{2}$ Departamento de Engenharia Florestal, Universidade Federal do Paraná, Curitiba, PR, Brasil. \\ *E-mail: veronica.kazama@gmail.com
}

Recebido em março/2018; Aceito em junho/2018.

\begin{abstract}
RESUMO: Esta pesquisa objetivou analisar a eficiência do carbono sequestrado em diferentes classes de uso e ocupação da terra entre os anos de 1989 a 2016 do município de Lucas do Rio Verde, Mato Grosso, situado na fronteira sul da Amazônia. Foram utilizadas duas imagens orbitais da série Landsat (TM e OLI), ambas do mês de julho. Efetuou-se a seleção do melhor método de classificação do uso e ocupação da terra, testando-se algumas composições de bandas. A estimativa da eficiência do carbono sequestrado foi efetuada pelo índice $\mathrm{CO}_{2 \mathrm{f}}$ flux. Para a classificação das categorias, foi selecionado o método de Máxima Verossimilhança, com composição de bandas: infravermelho médio 1, infravermelho próximo e vermelho. Em 27 anos, a área classificada como agricultura obteve um aumento de $172,90 \%$, sendo a vegetação nativa a mais afetada $(-45,26 \%)$ em perda de área. Neste período, identificou-se uma redução da média da eficiência do sequestro de carbono da vegetação nativa $(48,48 \%)$ e da área total $(52,17 \%)$, que ocorreu devido a perda da cobertura nativa, na qual consiste na cobertura de maior atividade fotossintética, portanto maior carbono fixado. Conclui-se que as alterações do uso da terra impactam na eficiência do sequestro de carbono da área de estudo no período analisado.
\end{abstract}

Palavras-chave: análise temporal, $\mathrm{CO}_{2 \mathrm{e}}$ flux, classificação digital de imagens.

\section{Impact of land use on the efficiency of carbon sequestry in the south of the Amazon}

\begin{abstract}
The objective of this research was to analyze the efficiency of the sequestered carbon in different land use cover between 1989 and 2016 in the municipality of Lucas do Rio Verde, Mato Grosso, located at the southern border of the Amazon. Two orbital images of the Landsat series (TM and OLI) were analyzed, both from July. The best classification method of land use and occupation was selected, and some band compositions were tested. The assessment of the efficiency of the sequestered carbon was made by the $\mathrm{CO}_{2 e f l u x}$ index. For the classification of the classes, the selected method was maximum likelihood, with composition of bands: medium infrared, near infrared and red. In 27 years, the area classified as agriculture increased by $172.90 \%$, and the native vegetation was the most affected $(-45.26 \%)$ in area loss. The work identified a reduction in the average of the carbon sequestration efficiency of native vegetation $(48.48 \%)$ and the total area $(52.17 \%)$, which occurred due to the loss of native vegetation, in which it consists of the coverage of greater photosynthetic activity, therefore greater fixed carbon. The study concludes that changes in land use impact on the carbon sequestration efficiency of the study area in 27 years.
\end{abstract}

Keywords: temporal analysis, $\mathrm{CO}_{2 \mathrm{e}}$ flux, digital classification of images.

\section{INTRODUÇÃO}

Nas últimas décadas, a Amazônia brasileira tem sofrido fortes alterações no uso e ocupação da terra (GALFORD et al., 2010), principalmente sua porção sul pelo avanço da agricultura, impulsionada pela demanda do mercado, global e nacional, de alimentos, fibras e de combustível (NEPSTAD et al., 2008).

Os desmatamentos e queimadas da cobertura vegetacional, para permitir os diversos usos antrópicos, são mudanças que afetam os processos biogeoquímicos e biogeofísicos do ambiente que podem intensificar as emissões de gazes de efeito estufa (GEEs). Durante o desmatamento, o carbono é perdido por meio do processo de corte e queima da biomassa, sendo $1,1 \%$ liberado como metano $\left(\mathrm{CH}_{4}\right)$ e o restante em forma de dióxido de carbono $\left(\mathrm{CO}_{2}\right)$ (GALFORD et al., 2010).

Além disso, as modificações na cobertura vegetacional alteram as trocas de fluxos de diversos elementos entre a atmosfera e superfície da terra. Em decorrência disso, são afetados os ciclos biogeoquímicos, como o da água, carbono nitrogênio e fósforo, bem como, afetam as variáveis biofísicas como o albedo, eficiência da evapotranspiração e rugosidade da superfície, nas quais são fatores responsáveis pela alteração do clima (DAVIN; NOBLET-DUCOUDRÉ, 2010).

As consequências climáticas devido aos GEEs emitidos por áreas de produção agrícola são preocupações de diversas pesquisas, como pode-se citar os trabalhos de Galford et al., (2010), que estimou, no contexto de um clima futuro, os impactos da dinâmica dos GEEs dos ecossistemas naturais e ecossistemas agrícolas após o desmatamento; Yue et al. (2017), que avaliou os impactos climáticos de uma variedade de cultivos e produtos agrícolas, desde a produção até o consumo; e Pradhan et al. (2017), que identificou as medidas economicamente atraentes para reduzir as emissões de GEEs do setor de agricultura, silvicultura e outros usos da terra conforme diferentes preços do carbono.

Em geral, os autores citados convergem sobre a necessidade de, além da conscientização do hábito de consumo alimentar (YUE et al., 2017; PRADHAN et al., 2017), avaliar 
os objetivos do desenvolvimento agrícola com a sustentabilidade ambiental no planejamento e gestão do uso e ocupação da terra, como uma forma de mitigação das emissões de GEEs, especialmente, nas regiões situadas na fronteira sul da Amazônia brasileira (GALFORD et al., 2010).

Dentre as áreas de expansão agrícola nessa região da Amazônia, o estado de Mato Grosso (MT) tem destaque por ser líder na produção nacional de grãos (IBGE, 2017). No entanto, este cenário traz como consequência o aumento das emissões dos GEEs no Estado, pois desde 1990 a mudança de uso da terra e floresta, devido aos desmatamentos, é considerada a maior fonte de emissões de gás carbônico $\left(\mathrm{CO}_{2}\right)$ (SEEG BRASIL, 2018).

Por meio de dados e técnicas de sensoriamento remoto é possível estimar índices espectrais que permitem estimar indiretamente a distribuição do carbono fixada em uma superfície da terra. Como é o caso do índice de $\mathrm{CO}_{2 \mathrm{e}}$ flux, que avalia eficiência do sequestro de carbono de uma cobertura vegetacional fotossinteticamente ativa (BAPTISTA, 2004).

$\mathrm{O}$ índice de $\mathrm{CO}_{2 \mathrm{e}}$ flux é calculado pela integração de dois índices espectrais, o NDVI (Normalized Difference Vegetation Index) e o PRI (Photosynthetic Reflectance Index) utilizando imagens orbitais. O NDVI analisa a absorção da radiação à fotossíntese de uma vegetação em vigor e o PRI mede a utilização da radiação no processo de fotossíntese da vegetação verde (RAHMAN et al., 2000, BAPTISTA, 2004).

No Brasil, este índice tem sido utilizado por diversos estudos ambientais como, por exemplo, em áreas de queimadas (TEOBALDO; BAPTISTA, 2016), pastagens e cobertura vegetacional (SILVA; BAPTISTA, 2015).

O objetivo do presente estudo é realizar uma análise temporal, para os anos de 1989 e 2016, da eficiência do sequestro de carbono em diferentes classes de uso e ocupação da terra, por meio da classificação supervisionada de imagens orbitais, do satélite Landsat 5 e 8, para o município de Lucas do Rio Verde, MT.

\section{MATERIAL E MÉTODOS}

\section{1. Área de estudo}

Para realizar o presente estudo foi selecionado o município de Lucas do Rio Verde, localizado na região Médio Norte do estado de Mato Grosso, que está situado no Centro-Oeste do Brasil (Figura 1). A escolha do município justifica-se por ser um dos maiores produtores de soja, com uma produção estimada de três toneladas por hectare, situado na fronteira sul da Amazônia Legal (IBGE, 2017).

O município ( $13^{\circ} 04^{\prime} 33^{\prime \prime} \mathrm{S}, 55^{\circ} 44^{\prime} 41^{\prime}$ 'W e 400 metros de altitude) apresenta uma extensão territorial de $3.661,83 \mathrm{~km}^{2}$, localizado no meio-norte do Estado, em uma região de Floresta de Transição Amazônia Cerrado. Tem como base econômica a agricultura em larga escala, incluindo a produção de soja, algodão, arroz e milho, além de aves e suínos (IBGE, 2006).

\subsection{Obtenção e Processamento dos dados}

Para este estudo utilizou-se: a base cartográfica digital abrangendo a área de estudo, adquiridas no portal do Instituto Brasileiro de Geografia e Estatística - IBGE (https://www.ibge.gov.br/); uma imagem do satélite Landsat 5, sensor TM (Thematic Mapper) datado de 21 de julho de 1989 e um outra imagem do satélite Landsat 8, sensor OLI (Operational Land Imager) de 31 de julho de 2016. Ambas as imagens de satélite foram adquiridas gratuitamente pelo catálogo de imagens do Instituto Nacional de Pesquisas
Espaciais - INPE (http://www.inpe.br/), com órbita/ponto 227/69 e bandas multiespectrais de resolução espacial de 30 metros.

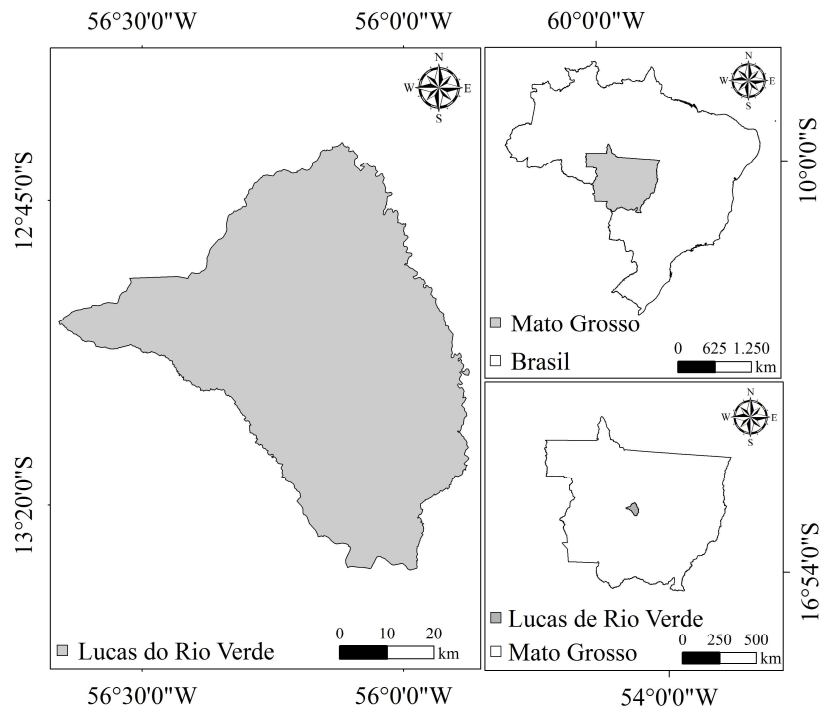

Figura 1. Localização da área de estudo. Figue 1. Location of study área.

As imagens foram adquiridas nessas datas por serem referentes ao período de seca da região, que ocorre entre maio e setembro, tendo como pico máximo de deficiencia hídrica em julho (SOUZA et al., 2013). Este é o período climático em que os plantios agrícolas na região Médio Norte de MT são colhidos e/ou em preparação do solo para o próximo cultivo (IMEA, 2018). Além disso, esse período apresenta como vantagem a ausência de nuvens que poderiam interferir na análise dos dados.

Foi realizada a correção geométrica entre a imagem Landsat 5 em relação à imagem Landsat 8. Para tal, os pontos de controle foram coletados de maneira bem distribuída pela extensão da imagem, de maneira a obter um Erro Quadrático Médio (RMS) menor que meio $(0,5)$ pixel.

Em seguida, os números digitais dos pixels da imagem foram convertidos em radiância e realizada a correção atmosférica para a obtenção dos valores de reflectância de superfície na imagem. Nesta etapa foi utilizado o algoritmo FLAASH (Fast Line-of-Sight Atmospheric Analysis of Spectral Hypercubes) que corrige os efeitos do espalhamento e absorção atmosférica baseado no modelo de transferência radiativa MODTRAN (EXELIS, 2014). As imagens corrigidas foram recortadas conforme o limte do municipio.

\subsection{Classificação de imagens}

Para a classificação digital das imagens foram definidas as seguintes classes de uso e ocupação do solo: i) hidrografia: rios, lagos e lagoas naturais e artificiais; ii) vegetação nativa: formação arbórea, arbustiva e campestre que são presentes tanto no bioma Amazônia quanto no Cerrado; iii) agricultura: áreas antrópicas agrícolas com cobertura conforme a classificação do IBGE (2013) que abrange nessa categoria as lavouras temporárias, lavouras permanentes, silvicultura e pastagens plantadas; iv) solo exposto: áreas sem cobertura vegetacional e abrange afloramento rochoso; v) queimada: áreas onde sofreram a ação do fogo que possuem carvão e 
cinzas advindos da carbonização da cobertura presente posteriormente a passagem do fogo.

Em seguida, a classificação digital das imagens foi realizada de forma supervisionada, sendo testado o desempenho de dois algoritmos (etapa 1): Distância Mínima (DM) e Máxima Verossimilhança (MaxVer), escolhidos pela facilidade de parametrização e execução (ANTUNES et al., 2012). Sendo utilizadas todas as bandas para seleção do melhor algoritmo.

Foram coletadas amostras de treinamento para cada classe de uso da terra, por meio de análise visual das feições da cobertura e uso da terra. Foram utilizadas as bandas do visível $(1,2,3)$ e do infravermelho próximo e médio $(4,5$ e 7$)$ do Landsat 5 para determinar o melhor algoritmo de classificação.

Em seguida, foi testado o desempenho de diferentes composições de bandas (etapa 2). Em uma das composições foi utilizado um índice de vegetação, o NDVI, ainda na imagem Landsat 5, para no intuito de se obter um melhor resultado. O uso do NDVI é justificado por permitir a redução da dimensionalidade dos dados em uma banda, bem como, uma melhor diferenciação das classes por utilizar a razão de bandas (vermelho e infravermelho próximo) que auxilia na distinção das superfícies com vegetação (BAPTISTA 2004).

O NDVI (Equação 1) consiste na diferença de reflectância entre a feição de absorção do vermelho $(0,66 \mu \mathrm{m})$ e o aumento de albedo que ocorre nos espectros de vegetação logo após ao início do infravermelho próximo $(0,80 \mu \mathrm{m})$ (BAPTISTA, 2004). Este permite analisar a vegetação fotossinteticamente ativa pela radiação absorvida pela mesma. Os valores variam de 1 a -1 , sendo quanto mais próximo de 1 , maior a densidade da cobertura vegetal e os valores menores ou iguais a zero corresponde a ausência de vegetação na superfície (RAHMAN et al., 2001).

$$
N D V I=\frac{I V P-R}{I V P+R}
$$

(Equação 1)

em que: IVP = reflectância no infravermelho próximo; RED = reflectância no vermelho.

Um resumo da metodologia para selecionar a melhor algoritmo de classificação e composição de bandas para imagem Landsat 5 é demostrada na Tabela 1. Em sequência, a melhor composição de bandas para o melhor algoritmo classificador para imagem Landsat 5, foi reaplicada na imagem do satélite Landsat 8 (etapa 3).

Tabela 1. Composição de bandas utilizadas para a classificação digital de imagens de Lucas do Rio Verde, MT.

Table 1. Composition of bands used for the digital classification of images of Lucas do Rio Verde, MT.

\begin{tabular}{cccc}
\hline ET & IL & Classificador & Composição de Bandas \\
\hline 1 & 5 & DM & TaxVer \\
\hline 2 & 5 & $\begin{array}{c}\text { Melhor algoritmo da etapa as bandas } \\
1\end{array}$ & $\begin{array}{c}\text { IVM1- IVP- RED } \\
\text { NDVI- IVM1- IVM2 }\end{array}$ \\
\hline 3 & 8 & $\begin{array}{c}\text { Melhor algoritmo no } \\
\text { Landsat 5 }\end{array}$ & $\begin{array}{c}\text { Melhor composição no } \\
\text { Landsat 5 }\end{array}$ \\
\hline
\end{tabular}

ET = etapa; $\mathrm{IL}$ = imagem Landsat; DM = distância mínima; MaxVer = máxima verossimilhança; $\mathrm{RED}=$ banda do vermelho; IVP = infravermelho próximo; IVM1 = infravermelho médio 1 ; IVM2 = infravermelho médio 2 ; NDVI = índice de vegetação normalizada.
Para a verificação da qualidade da classificação digital de imagens foi efetuada pela Matriz de Confusão, na qual a acuária e a concordância das classes foram averiguadas pelos índices de Exatidão Global (G, Equação 2) e de Kappa (K, Equação 3) (LANDIS; KOCH,1977).

$$
\begin{aligned}
& G=\frac{\sum_{i=1}^{M} n_{i i}}{N}=P_{0} \\
& K=\frac{P_{0}-P_{e}}{1-P_{e}}
\end{aligned}
$$

em que $n_{i i}=$ representa o número de pixels da diagonal principal da Matriz de Confusão de cada classe; $N=$ número de classes; $P_{0}=$ Concordância Total ou Exatidão Global; $P_{e}=$ concordância esperada.

Os índices foram ranqueados conforme o melhor desempenho das classifcações, sendo que, quanto mais próximo de 1 os valores de $\mathrm{G}$ e K, melhor são os resultados. Para o índice K, seguiu-se a classificação proporsta por Landis e Koch (1977) (Tabela 2).

Tabela 2. Classes de avaliação da qualidade da classificação do mapa temático quanto ao índice Kappa.

Table 2. Classes of evaluation of the quality of the classification of the thematic map regarding the Kappa index.

\begin{tabular}{cc}
\hline Valor de Kappa & Qualidade da classificação \\
\hline$<0,00$ & Péssima \\
$0,00 \mid-0,20$ & Ruim \\
$0,20 \mid-0,40$ & Razoável \\
$0,40 \mid-0,60$ & Boa \\
$0,60 \mid-0,80$ & Muito Boa \\
$0,80 \mid-1,00$ & Excelente \\
\hline
\end{tabular}

Fonte: Adaptado de Landis e Koch (1977)

\subsection{Estimativa do $\mathrm{CO}_{2 \mathrm{e}}$ flux}

A eficiência do sequestro de carbono pode ser medida pelo índice de $\mathrm{CO}_{2 \mathrm{e}}$ flux, que integra os índices NDVI e o PRI (RAHMAN et al., 2000).

O PRI (Equação 4), consiste na diferença existente entre a feição de absorção na transição do azul para o verde $(0,531$ $\mu \mathrm{m})$ e o pico de reflectância do verde $(0,570 \mu \mathrm{m})$. É um índice que se correlaciona a eficiência do uso da luz no processo de fotossíntese ocorrido na planta (SILVA; BAPTISTA, 2015). Os valores variam entre -1 e 1 , e valores comuns para vegetação sadia ficam entre -0,2 e 0,2 (BAPTISTA, 2004). O PRI tem necessidade de ser reescalonado em valores positivos para se adequar à escala do NDVI (RAHMAN et al., 2001), assim, gerou-se um novo índice sPRI (Equação 5) que varia de 0 a 1 .

$$
\begin{aligned}
& P R I=\frac{B L U E-G R E E N}{B L U E+G R E E N} \\
& S P R I=\frac{P R I}{2}+1
\end{aligned}
$$

em que: GREEN = é a reflectância no verde; BLUE = reflectância no azul.

A estimativa do índice $\mathrm{CO}_{2 \mathrm{e}}$ flux (Equação 6) é obtida pela multiplicação dos índices NDVI e sPRI.

$$
\mathrm{CO}_{2 e} \text { flux }=N D V I * s P R I
$$

(Equação 6)

$\mathrm{O}$ índice $\mathrm{CO}_{2 \mathrm{e}}$ flux mede a eficiência do sequestro de carbono pela vegetação fotossinteticamente ativa. Os valores mais elevados do $\mathrm{CO}_{2 \mathrm{e}}$ flux representam as áreas com maior atividade fotossintética, uma vez que são observadas maiores 
feições de absorção nas faixas do azul e do vermelho (RAHMAN et al., 2000; BAPTISTA, 2004).

\subsection{Análise temporal}

Para realizar a análise temporal da dinâmica do uso e cobertura da terra do município de Lucas do Rio Verde utilizou-se as imagens classificadas pelo melhor algoritmo e composição de bandas.

Há intervalo de tempo de 27 anos entre as imagens (19892016). As áreas que alteraram neste período foram identificadas e quantificadas em hectares e porcentagem. As delimitações das áreas dos diferentes usos e ocupação da terra foram utilizadas para identificar a eficiência do sequestro de carbono, pelo índice $\mathrm{CO}_{2 \mathrm{e}}$ flux, de cada classe de uso e ano, separadamente. A análise temporal da dinâmica do sequestro de carbono foi realizada por área total e por classe de uso e ocupação da terra para cada ano.

\section{RESULTADOS}

\subsection{Classificação de imagens}

O desempenho das classificações ranqueado conforme os critérios de precisão Exatidão Global $(\mathrm{G})$ e índice Kappa $(\mathrm{K})$ é mostrado na Tabela 3. Os valores mais próximos de 1 , destes índices, obtiveram-se colocações superiores devido à melhor precisão da classificação da imagem.

Os resultados mostram que o melhor algoritmo de classificação ao utilizar todas as bandas (etapa 1), na imagem Landsat 5, é dada pelo algoritmo $\operatorname{MaxVer}(\mathrm{G}=0,9986$ e $\mathrm{K}=$ 0,9350). Utilizando esse algoritmo no teste de melhor composição de bandas (etapa 2), ainda na imagem Landsat 5, a composição IVM1, IVP e RED obteve-se o melhor resultado $(\mathrm{G}=0,9940$ e $\mathrm{K}=0,9924)$.

O melhor algoritmo (MaxVer) e composição de bandas (IVM1, IVP e RED) obtidas na Landsat 5 foi utilizada na imagem Landsat 8 (etapa 3). A classificação da imagem Landsat 8 utilizando esta metodologia obteve-se também um bom desempenho $(\mathrm{G}=0,9981$ e $\mathrm{K}=0,9849)$ (Tabela 3).

3.2 Análise temporal da dinâmica do uso e ocupação da terra

As mudanças de uso e ocupação da terra em hectares e em percentual ocorridas no intervalo de 27 anos (1989-2016) são representadas em forma de matriz na Tabela 4, enquanto que na Figura 2 é mostrada espacialmente a distribuição destas mudanças.

Tabela 3. Desempenho da classificação por diferentes algoritmos e composição de bandas para as imagens orbitais do satélite Landsat 5 e Landsat 8, em Lucas do Rio Verde, MT.

Table 3. Classification performance by different algorithms and band composition for orbital images of Landsat 5 and Landsat 8 satellite, in Lucas do Rio Verde, MT.

\begin{tabular}{|c|c|c|c|c|c|c|}
\hline ET & IL & Classificador & $\begin{array}{l}\text { Composição de } \\
\text { Bandas }\end{array}$ & G & K & Ran \\
\hline 1 & 5 & $\begin{array}{c}\text { DM } \\
\text { MaxVer }\end{array}$ & Todas as bandas & $\begin{array}{l}0,9486 \\
0,9986 \\
\end{array}$ & $\begin{array}{l}0,9350 \\
0,9350 \\
\end{array}$ & $\begin{array}{l}2 \\
1 \\
\end{array}$ \\
\hline \multirow[b]{2}{*}{2} & \multirow[b]{2}{*}{5} & \multirow[b]{2}{*}{ MaxVer } & IVM1-IVP-RED & 0,9940 & 0,9924 & 1 \\
\hline & & & $\begin{array}{l}\text { NDVI- IVM1- } \\
\text { IVM2 }\end{array}$ & 0,9833 & 0,9787 & 2 \\
\hline 3 & 8 & MaxVer & IVM1-IVP-RED & 0,9881 & 0,9849 & - \\
\hline
\end{tabular}

ET= etapa; IL = imagem Landsat; G= Exatidão Global; K= índice Kappa; Ran = ranking; $\mathrm{DM}=$ Distância Mínima; MaxVer = Máxima Verossimilhança; RED = banda do vermelho; IVP = infravermelho próximo; IVM1 = infravermelho médio 1; IVM2 = infravermelho médio 2; NDVI = índice de vegetação normalizada.

A dinâmica de uso e ocupação da terra da área de estudo mostra que em 1989 a classe de maior predominância era a vegetação nativa com $41,77 \%(1.529,12 \mathrm{ha})$ da área total. Em 2016, foi alterada para agricultura ocupando 54,97\% (2.012,56 ha) da área total do município.

Em 1989, dentre as classes que sofreram alteração para 2016, a classe queimada foi a que teve a maior mudança $(99,22 \%)$ em área total, convertidos em sua maioria $(65,99 \%)$ para agricultura. A agricultura também foi a que apresentou o maior aumento percentual (172,90\%) em área (1.277,78 ha) no final do período. O solo exposto é o responsável pelo maior montante $(999,19$ hectares) e percentual (71,95\%) de mudanças para agricultura. Por outro lado, a vegetação nativa foi a classe que mais perdeu área entre o período $(-45,26 \%)$. Estas mudanças denotam o fato da agricultura ter tido um significativo aumento de área no período de tempo considerado (1.277,78 ha), o que corresponde a cerca de $172,90 \%$ no decorrer destes 27 anos de análise.

Tabela 4. Matriz de mudanças entre os anos de 1989 e 2016 para as diferentes classes de uso e ocupação da terra, em de Lucas do Rio Verde, MT.

Table 4. Matrix of changes between the years of 1989 and 2016 for the different land use and occupation classes, in Lucas do Rio Verde, MT.

\begin{tabular}{|c|c|c|c|c|c|c|c|}
\hline & \multirow{2}{*}{$\begin{array}{c}\text { Hectares } \\
(\%)\end{array}$} & \multicolumn{5}{|c|}{ Imagem de 1989 (Landsat 5) } & \multirow{2}{*}{$\begin{array}{c}\text { Total } \\
(2016) \\
\end{array}$} \\
\hline & & Solo exposto & Agricultura & Queimada & Hidrografia & Vegetação nativa & \\
\hline \multirow{5}{*}{$\begin{array}{c}\text { Imagem de } \\
2016 \\
\text { (Landsat 8) }\end{array}$} & Solo exposto & $\begin{array}{l}381,29 \\
(27,62)\end{array}$ & $\begin{array}{c}191,66 \\
(26)\end{array}$ & $\begin{array}{c}3,39 \\
(29,15)\end{array}$ & $\begin{array}{c}0,09 \\
(1,67)\end{array}$ & $\begin{array}{l}213,33 \\
(13,95)\end{array}$ & $\begin{array}{c}789,76 \\
(100)\end{array}$ \\
\hline & Agricultura & $\begin{array}{l}993,19 \\
(71,95)\end{array}$ & $\begin{array}{l}503,75 \\
(68,56)\end{array}$ & $\begin{array}{c}7,69 \\
(65,99)\end{array}$ & $\begin{array}{c}2,29 \\
(44,27)\end{array}$ & $\begin{array}{l}505,64 \\
(33,07)\end{array}$ & $\begin{array}{c}2.012,56 \\
(100)\end{array}$ \\
\hline & Queimada & $\begin{array}{c}0,58 \\
(0,04)\end{array}$ & $\begin{array}{c}10,2 \\
(1,39)\end{array}$ & $\begin{array}{c}0,09 \\
(0,78)\end{array}$ & $\begin{array}{c}0,07 \\
(1,34)\end{array}$ & $\begin{array}{c}2,80 \\
(0,18)\end{array}$ & $\begin{array}{l}13,74 \\
(100)\end{array}$ \\
\hline & Hidrografia & $\begin{array}{c}0,11 \\
(0,01)\end{array}$ & $\begin{array}{c}1,17 \\
(0,16)\end{array}$ & $\begin{array}{c}0,29 \\
(2,50)\end{array}$ & $\begin{array}{c}1,25 \\
(24,17)\end{array}$ & $\begin{array}{c}5,26 \\
(0,34)\end{array}$ & $\begin{array}{c}8,09 \\
(100)\end{array}$ \\
\hline & Vegetação nativa & $\begin{array}{r}5,31 \\
(0,38) \\
\end{array}$ & $\begin{array}{l}28,01 \\
(3,81) \\
\end{array}$ & $\begin{array}{c}0,18 \\
(1,58) \\
\end{array}$ & $\begin{array}{c}1,48 \\
(28,56) \\
\end{array}$ & $\begin{array}{l}802,08 \\
(52,45)\end{array}$ & $\begin{array}{c}837,06 \\
(100)\end{array}$ \\
\hline \multicolumn{2}{|l|}{ Total (1989) } & $1.380,48$ & 734,78 & 11,65 & 5,18 & $1.529,12$ & - \\
\hline \multicolumn{2}{|c|}{ Mudanças entre 1989 - 2016} & 999,19 & 231,03 & 11,55 & 3,93 & 727,04 & - \\
\hline \multicolumn{2}{|c|}{ Diferenças entre 1989 - 2016} & $-590,72$ & $1.277,78$ & 2,09 & 2,91 & $-692,06$ & - \\
\hline
\end{tabular}

Em que os valores nas colunas são relativos a área de 1989 e os valores nas linhas a de 2016. A diagonal principal da matriz de mudança mostra à extensão inalterada entre um ano para o outro. 

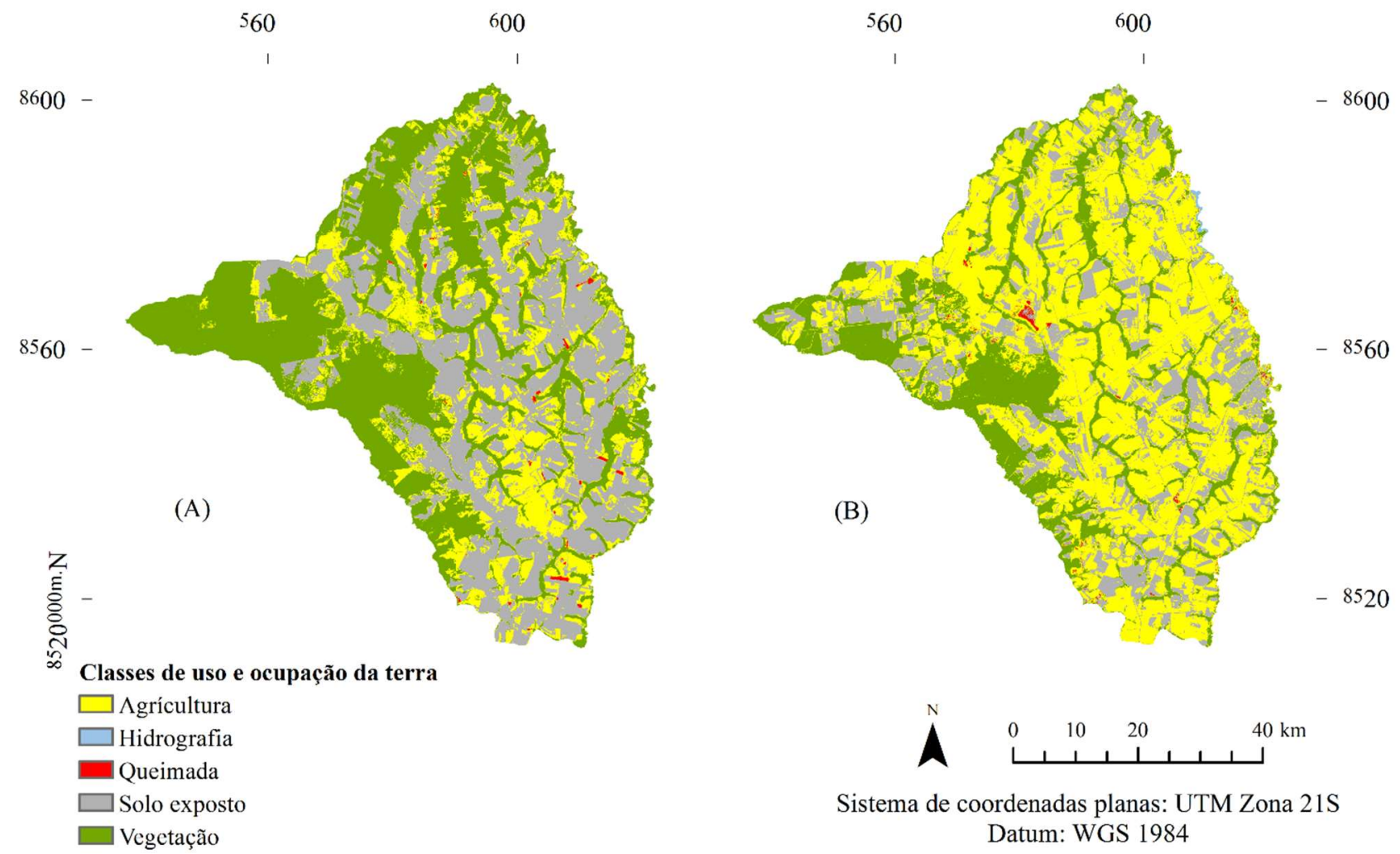

Sistema de coordenadas planas: UTM Zona $21 \mathrm{~S}$ Datum: WGS 1984

Figura 2. Dinâmica do padrão do uso e ocupação da terra de 1989 (A) e 2016 (B) em Lucas de Rio Verde, MT. Figure 2. Dynamics of land use and coverage of 1989 (A) and 2016 (B) in Lucas de Rio Verde, MT.

Além disso, a classe de agricultura foi a que apresentou menor mudança $(31,44 \%)$ para outro uso da terra no período. Em outras palavras, foi a classe que mais se manteve sem alteração $(68,56 \%)$ do total de área que possuía em 1989.

Já a classe de hidrografia mostrou-se como a de menor área, tanto em 1989 como em 2016, porém com um aumento em área de 56,11\% do ano de 1989 para 2016. O aumento das áreas de hidrografia é explicado pela construção da Usina hidrelétrica Canoa Quebrada, que entrou em operação no início de 2007 (CAPARROZ, 2010).

\subsection{Análise temporal da dinâmica do $\mathrm{CO}_{2 \mathrm{e}}$ flux}

A média e o desvio padrão dos valores do índice $\mathrm{CO}_{2 \mathrm{e}}$ flux em área total e em diferentes classes de uso e ocupação da terra em Lucas do Rio Verde, MT, no ano de 1989 e 2016 pode ser observada na Tabela 5.

Na Figura 3, é apresentada espacialmente a dinâmica do $\mathrm{CO}_{2 \mathrm{e}}$ flux sobre a área de estudo em cada ano. Para facilitar a comparação entre as duas imagens, padronizou-se uma única legenda apresentando seis classes distintas para os valores do $\mathrm{CO}_{2 \mathrm{f} f l u x}$ Os menores valores estão em vermelho, seguida em ordem crescente da cor laranja, amarela, verde claro, verde médio até o verde escuro.

Conforme o resultado do índice $\mathrm{CO}_{2 \mathrm{e}}$ flux, nota-se que em 1989, existe uma maior abrangência de diferentes classes do índice $\mathrm{CO}_{2 e}$ flux do que em 2016. O que reflete no maior desvio padrão dos valores índice $\mathrm{CO}_{2 \mathrm{e}}$ flux no primeiro ano $(0,16)$ do que no segundo $(0,05)$. Para área total, observa-se que a média do índice $\mathrm{CO}_{2 \mathrm{e}}$ flux reduziuse de 0,23 para 0,12 em 27 anos (Tabela 5).
Tabela 5. Estatística descritiva do índice de $\mathrm{CO}_{2 \mathrm{e}}$ flux na área total e nas diferentes classes de uso e ocupação do solo de Lucas do Rio Verde, MT, no ano de 1989 e 2016.

Table 5. Descriptive Statistics of $\mathrm{CO}_{2 \mathrm{e}}$ flux in the total area and in the different classes of land use and occupation of Lucas do Rio Verde, MT, in the years 1989 and 2016.

\begin{tabular}{lcccc}
\hline \multirow{2}{*}{ Classes } & \multicolumn{2}{c}{1989} & \multicolumn{2}{c}{2016} \\
\cline { 2 - 5 } & Média & $\begin{array}{c}\text { Desvio } \\
\text { Padrão }\end{array}$ & Média & $\begin{array}{c}\text { Desvio } \\
\text { Padrão }\end{array}$ \\
\hline Vegetação nativa & 0,41 & 0,06 & 0,20 & 0,01 \\
Solo exposto & 0,06 & 0,06 & 0,08 & 0,02 \\
Hidrografia & 0,15 & 0,10 & $5,3 \mathrm{e}^{-04}$ & 0,01 \\
Queimada & 0,01 & 0,02 & 0,13 & 0,01 \\
Agricultura & 0,10 & 0,06 & 0,12 & 0,04 \\
\hline Área Total & 0,23 & 0,16 & 0,12 & 0,05 \\
\hline
\end{tabular}

Entre as classes de uso e ocupação da terra existe uma grande distinção da eficiência do sequestro de carbono, onde os valores mais elevados do $\mathrm{CO}_{2 \mathrm{e}}$ flux estão sobre as áreas com maior atividade fotossintética. Em geral, conforme esperado, as maiores médias estão sobre as áreas de vegetação nativa, e as menores médias variam sobre as áreas de queimada (1989) e hidrografia (2016) (Tabela 5).

A classe de vegetação nativa também teve decréscimo em valores médios $(0,41)$ em 1989 para ano de $2016(0,20)$, assim como a classe de hidrografia. No entanto a hidrografia apresentou um alto valor em 1989 (0,15), acima do esperado para as características da classe e ainda mostrou o maior desvio padrão (10). Por outro lado, a área da agricultura apresentou um aumento na média de $\mathrm{CO}_{2 \mathrm{e}}$ flux de 0,10 para 0,12 , mostrando que o sequestro de carbono na área de agricultura aumentou (Tabela 5).

Em 2016, observa-se que as áreas agrícolas irrigadas, caracterizada pela forma geométrica circular, apresentam 
valores de $\mathrm{CO}_{2 \mathrm{e}}$ flux dentro de um intervalo $(0,21$ a 0,30$)$ superior ao comparado do intervalo da maior parte da área de agricultura $(0,07$ a 0,12$)$ e da vegetação nativa $(0,13$ a 0,20$)$
(Figura 3b), devido o menor estresse hídrico e assim melhor atividade vegetativa.
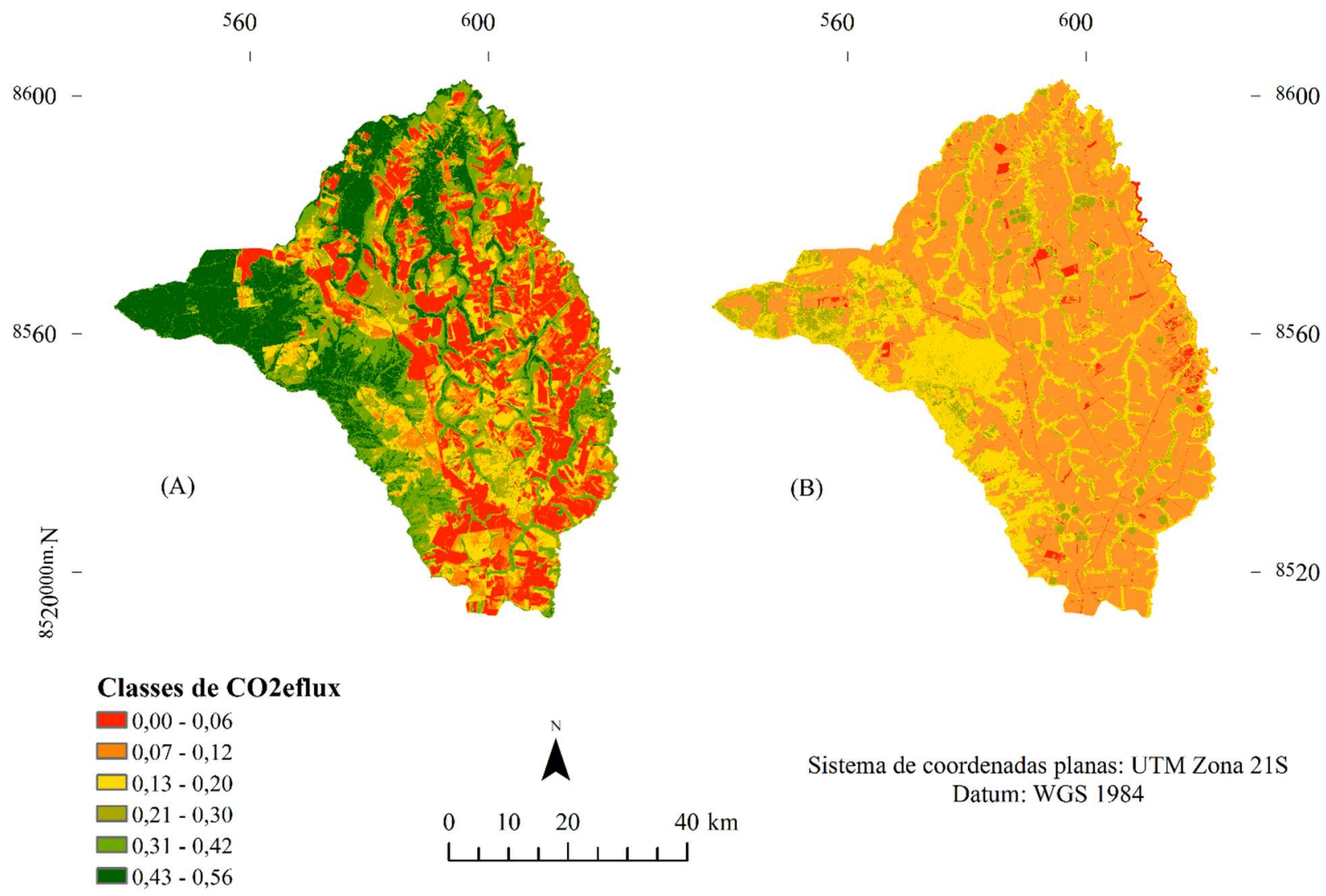

Sistema de coordenadas planas: UTM Zona 21S Datum: WGS 1984

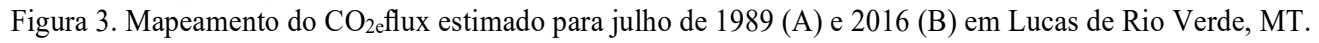
Figure 3. Mapping of the estimated $\mathrm{CO}_{2 \mathrm{e}}$ flux for July 1989 (A) and 2016 (B) in Lucas de Rio Verde, MT.

\section{DISCUSSÃO}

4.1 Classificações de imagens

O algoritmo MaxVer selecionado como melhor desempenho (Tabela 3) apresenta a classificação excelente conforme as categorias de avaliação qualitativa quanto ao índice Kappa, proposto por Landis e Koch (1977) (Tabela 1). Este algoritmo já foi selecionado em outros trabalhos como sendo o de melhor resultado pelo mesmo índice (JUNIATI; ARROFIQOH, 2017; MELLO et al., 2012)

A seleção da composição de bandas, infravermelho médio 1, infravermelho próximo e vermelho (IVM1-IVP-RED), pode ser explicada pelo fato dessa composição abranger a faixa espectral em que os alvos (classes de uso e ocupação) possuem uma maior reflectância (NOVO, 2011). Desta forma, a composição permitiu uma melhor distinção pelo o algoritmo classificador, resultando em um melhor desempenho. Além disso, por tais características, esta composição selecionada é usualmente utilizada em estudos de análise das feições de cobertura florestal, agrícolas desmatamentos, áreas degradadas e entre outros (ESPÍRITO-SANTO et al., 2005; RIZZI; RUDORFF, 2005)

4.2 Análise temporal da dinâmica do uso e ocupação da terra

A compreensão da dinâmica das alterações da cobertura de uma determinada superfície de área e em um determinado período são informações relevantes para auxiliar em uma análise espacial.
Conforme os resultados das mudanças de áreas do presente estudo (Tabela 4), é possível observar que a conversão de áreas queimadas e vegetação nativa para área de agricultura, em 27 anos, reflete um cenário comum do avanço do desmatamento de áreas nativas da Amazônia e Cerrado do Mato Grosso para o cultivo agrícola e a pecuária (GALFORD et al., 2010).

O histórico de desenvolvimento da tecnologia de cultivo agrícola de espécies anuais no Brasil mostra que a partir da década de 90, o sistema de plantio convencional (SPC) foi fortemente substituído pelo sistema de plantio direto (SPD) no país. Na qual, o SPD consiste em uma técnica de cultivo conservacionista, onde o solo é mantido sempre com uma cobertura de plantas e por resíduos vegetais para proteger o solo das gotas de chuva contra do escorrimento superficial e das erosões hídrica e eólica. O SPD é a modalidade do cultivo mínimo, onde o preparo do solo limita-se ao sulco de semeadura (EMBRAPA, 2011).

Considerando que o SPC apresenta uma fase em que o solo permanece sem cobertura, na etapa de preparo do solo, e o SPD, sempre apresenta uma cobertura de plantas em desenvolvimento e/ou por resíduos vegetais da última cultura plantada, pode ser que essa diferença tenha sido detectada na classificação digital de imagens da área da agricultura em 1989.

Assim, é possível considerar que algumas das áreas de solo exposto podem pertencer as áreas de cultivo agrícola do SPC, em 1989. Isto explica a grande mudança da classe de solo 
exposto para agricultura. E ainda, significa que a área de agricultura pode ser maior do que o calculado para 1989.

$\mathrm{O}$ fato da classe de agricultura ter mantido a maior área sem alteração, durante 27 anos, revela que a atividade econômica da agricultura permanece predominante na região, sendo crescente neste período e tendendo a continuar em crescimento nos próximos anos. São diversos os fatores favoráveis para este cenário, dentre estes destacam-se o relevo plano e o regime pluviométrico que ocorre na região, permitindo que seja colhido duas safras por ano nesta região, diferente da região Leste e Sudeste do MT (ARVOR et al., 2017).

A dinâmica do uso da terra em Mato Grosso é considerada complexa e representa uma variedade de caminhos que conduzem ao estabelecimento de terras cultivadas (GALFORD et al., 2010). Os resultados do presente trabalho convergem para tal afirmação citada.

Apesar disso, atualmente o município não se encontra na lista de cidades do MT prioritárias para ações de prevenção, monitoramento e controle do desmatamento (MMA, 2017). Um do motivo se deve ao fato do município estar situada na fronteira consolidada do desmatamento na Amazônia ou "fronteira fechada", onde o desmatamento foi mais intenso na década de 70 (WANDERLEY, 2018). Mesmo assim, é necessário um monitoramento constante para detecção de irregularidades ambientais quanto ao desmatamento ilegal na região, pois essas mudanças no uso da terra são responsáveis pela maior parcela de emissão de $\mathrm{CO}_{2}$ na atmosfera no estado do MT (SEEG BRASIL, 2018).

\subsection{Análise temporal da dinâmica do $\mathrm{CO}_{2 \mathrm{e}}$ flux}

Com o uso do sensoriamento remoto é possível relacionar indiretamente quanto de $\mathrm{CO}_{2}$ é sequestrado pela biomassa em determinada superfície terrestre, por meio do índice de $\mathrm{CO}_{2 \mathrm{e}}$ flux proposto por Rahman et al. (2000). Uma vez que integra índices espectrais, como o NDVI, que possibilita verificar a vegetação fotossinteticamente ativa, e PRI, que analisa a eficiência do uso da luz no processo de fotossíntese (SILVA; BAPTISTA, 2015).

É de conhecimento que a perda de água na vegetação implica na redução da absorção das feições do azul e do vermelho, que são verificados pelo NDVI e PRI. Neste sentido, quanto menor a atividade fotossintética da vegetação, menor é absorção nessas faixas do espectro o que resulta em menor valor do $\mathrm{CO}_{2 \mathrm{e}}$ flux (BAPTSITA, 2004).

$\mathrm{O} \mathrm{CO}_{2 \mathrm{e}}$ flux pode ser correlacionado com a concentração e o fluxo de $\mathrm{CO}_{2}$ da atmosfera coletadas em torres micrometeorológicas, visto que Silva e Baptista (2015), utilizando dados de uma torre instalada em diferentes ambientes na Amazônica brasileira, encontraram que o comportamento do índice $\mathrm{CO}_{2 \mathrm{e}}$ flux, estimada com dados do sensor Hyperion, possui uma forte correlação negativa com a concentração $(\mathrm{ppm})$ e fluxo $\left(\mathrm{mol} \cdot \mathrm{m}^{-2} \cdot \mathrm{s}^{-1}\right)$ de $\mathrm{CO}_{2}$, pelo teste de Pearson.

Considerando os resultados dos autores supracitados, apesar do presente trabalho não contar com dados de concentração e fluxo de $\mathrm{CO}_{2}$ coletados em campo, permite-se analisar que quanto maior for a atividade fotossintética medida pelo $\mathrm{CO}_{2 \mathrm{e}}$ flux, menor é a concentração (ppm) e fluxo de $\mathrm{CO}_{2}$ $\left(\mathrm{mol} \cdot \mathrm{m}^{-2} \cdot \mathrm{s}^{-1}\right)$, pois o $\mathrm{CO}_{2}$ estaria fixado na vegetação reduzindo o na atmosfera.

Este fato corrobora com Baptista (2004) ao afirmar que áreas com maior densidade florestal, como as matas ciliares, tendem a apresentar valores elevados de eficiência de sequestro de carbono e baixos teores de $\mathrm{CO}_{2}$ atmosférico, uma vez que quanto maior o sequestro, menos $\mathrm{CO}_{2}$ na atmosfera existirá na coluna sobre o alvo.

Ao observar os resultados obtidos na estimativa do sequestro de carbono, pelo $\mathrm{CO}_{2 \mathrm{e}}$ flux, na área de estudo do presente trabalho (Tabela 5), verifica-se que em média os valores obtidos do presente trabalho se encontram dentro da variação, entre 0,15 a 0,45 do índice $\mathrm{CO}_{2 \mathrm{e}}$ flux, estimadas por meio de dados de fluxo $\left(\mathrm{mol} \cdot \mathrm{m}^{-2} \cdot \mathrm{s}^{-1}\right)$ coletados em torres micrometeorológicas em diferentes ambientes na Floresta Amazônica (SILVA; BAPTISTA, 2015).

A redução da média de $\mathrm{CO}_{2 \mathrm{e}}$ flux da área de vegetação nativa entre o período analisado, pode estar relacionado ao fato de que os remanescentes florestais se apresentaram em menores áreas, em 2016 (Figura 3). Desta forma, pressupõe-se que esses remanescentes há 27 anos atrás, se apresentavam em uma melhor condição de preservação e, consequentemente, um melhor desenvolvimento das atividades fotossintéticas, pois quanto maior o valor de $\mathrm{CO}_{2 \mathrm{e}}$ flux (TEOBALDO; BAPTISTA, 2016), maior quantidade de carbono estocado na vegetação, o que indica melhores condições de desenvolvimento para a mesma (GRILO et al., 2011).

No entanto, observa-se que nessas as áreas agrícolas irrigadas (2016) apresentaram valores de $\mathrm{CO}_{2 \mathrm{e}}$ flux dentro de um intervalo superior do que o da vegetação nativa, como pode ser observado por sua coloração verde claro em relação a coloração amarela da maior parte da área de vegetação nativa. Isto ocorre pelo fato da área irrigada não estar sofrendo com o estresse hídrico do período de seca na região, e mantendo-se em plena atividade fotossintética.

Já nas áreas classificadas como queimadas, observa-se que o $\mathrm{CO}_{2 \mathrm{e}}$ flux assemelha-se com a área caracterizada como de pós-fogo do estudo de Teobaldo e Baptista (2016) no cerrado. Neste sentido, significa que os focos de queimadas na região de Lucas de Rio Verde ocorreram em um período anterior as datas da aquisição das imagens Landsat (TM e OLI) para efetuar o presente estudo, que foram dia 21 e 31 de julho.

Para a classe de hidrografia espera-se valores nulos de $\mathrm{CO}_{2 \mathrm{e}}$ flux devido a ausência de vegetação ou extremante valores baixos pela mistura do pixel com a vegetação de mata ciliar, que ocorre normalmente em uma classificação. Porém em 1989, mostrou-se uma alta média de $\mathrm{CO}_{2 \mathrm{e}}$ flux na classe de hidrografia. Esta situação pode estar relacionada aos erros de amostragem e classificação do algoritmo utilizado, em que, pode ter classificado certos pixels que não se constituíam de água e sim de algum tipo de vegetação. Esse erro influencia em uma maior variação entre os dados, o que faz compreender o maior desvio padrão $(0,10)$ dos valores de $\mathrm{CO}_{2 \mathrm{e}}$ flux da área da hidrografia em 1989.

Ao comparar o resultado da análise temporal da dinâmica do uso e ocupação da terra (Tabela 4) com o resultado da análise temporal da dinâmica do sequestro de carbono pelo $\mathrm{CO}_{2 \mathrm{e}}$ flux (Tabela 5), tem-se que a redução da área de vegetação nativa pode ser correlacionada com a redução da média do sequestro de carbono em área total, em 27 anos. Visto que a vegetação nativa é a classe de maior densidade de vegetação fotossinteticamente ativa, evidenciada pela maior média de $\mathrm{CO}_{2 \mathrm{e}}$ flux em ambas as imagens.

As áreas agrícolas obtiveram um leve aumento na média de $\mathrm{CO}_{2 \mathrm{e}}$ flux em 27 anos. Esse resultado, possivelmente tem relação com a transição da classe SPC para SPD da área de agricultura, que foi observado na análise da dinâmica do uso e 
ocupação da terra, entre os anos. Pois, agricultura em cultivo conservacionista promove o sequestro de carbono e tende a apresentar maiores valores de $\mathrm{CO}_{2 \mathrm{e}}$ flux.

A conversão de uma vegetação nativa para área agrícola utilizando SPC (como provavelmente era efetuado em 1989 no presente estudo) fomenta o esgotamento do carbono orgânico do solo, devido a redução de matéria orgânica do solo causado pelo revolvimento continuo, o que agrava a destruição de agregados (CHURCHMAN et al., 2010).

Já na agricultura conservacionista, por ser associada a alta e diversa entrada de carbono, restaura na agregação do solo e assim acumula carbono orgânico no solo ao longo do tempo (SÁ et al., 2015), apesar de ser menor ao carbono estocado, quando comprado com a cobertura florestal.

A conversão da agricultura com SPC para SPD observada, entre 27 anos, é destacado como um importante cenário do avanço tecnológico para a sustentabilidade da gestão agrícola da fronteira sul da Amazônia por proporcionar o aumento do sequestro do carbono no solo.

A quantificação da dinâmica do espaço-tempo associada à expansão das terras cultivadas à mudança dos padrões de cultivo é fundamental para muitas áreas de avaliação ecológica sustentabilidade agrícola, incluindo estimativas melhoradas de emissões de gases de efeito estufa, modelagem climática regional, ciclismo hidrológico, monitoramento da biodiversidade e fertilidade agrícola (GALFORD et al., 2010).

\section{CONCLUSÕES}

O melhor desempenho da classificação para gerar as áreas da análise temporal do uso e ocupação da terra e do sequestro de carbono, é selecionado o algoritmo MaxVer com a composição das bandas IFM1-IVP-RED;

A classe de maior área total em 1989 é a vegetação nativa, e em 2016 foi alterada para a agricultura. A área de agricultura apresenta-se com a menor área de mudanças para outras classes e com o maior aumento em área devido a mudança de outras classes para a mesma, em 27 anos;

$\mathrm{Na}$ dinâmica do sequestro de carbono pelo índice $\mathrm{CO}_{2 \mathrm{e}} \mathrm{flux}$, os valores médios em área total são reduzidos em decorrência do desmatamento da vegetação nativa para cultivo agrícola, no período analisado;

O uso de imagens orbitais e geoprocessamento são ferramentas relevantes para obter informações precisas com menor tempo de processamento para alcançar o objetivo do presente trabalho. Sendo indispensável para monitoramento das atividades antrópicas não só na fronteira sul da Amazônia, e sim em toda sua extensão, por esta ser constituído de extensas áreas.

\section{AGRADECIMENTOS}

O presente trabalho foi realizado com apoio da Coordenação de Aperfeiçoamento de Pessoal de Nível Superior - Brasil (CAPES) - Código de Financiamento 001 e do Conselho Nacional de Desenvolvimento Científico e Tecnológico (CNPQ), mediante a bolsa de estudos dos autores Pós-graduandos.

\section{REFERÊNCIAS}

ANTUNES, J. F. G.; MERCANTE, E.; ESQUERDO, J. C. D. M.; DE CAMARGO LAMPARELLI, R. A.; ROCHA, J. $\mathrm{V}$. Estimativa de área de soja por classificação de imagens normalizada pela matriz de erros. Pesquisa Agropecuária
Brasileira, v.47, n.9, p.1288-1294, 2012. DOI: https://doi.org/10.1590/s0100-204x2012000900014

ARVOR, D.; TRITSCH, I.; BARCELLOS, C.; JÉGOU, N.; DUBREUIL, V. Land use sustainability on the SouthEastern Amazon agricultural frontier: Recent progress and the challenges ahead. Applied Geography, v. 80, p. 8697, 2017.2 DOI: https://doi.org/10.1016/j.apgeog.2017.02.003

BAPTISTA, G. M. Mapeamento do sequestro de carbono e de domos urbanos de $\mathrm{CO}_{2}$ em ambientes tropicais, por meio de sensoriamento remoto hiperespectral. Geografia, v.29, n.2, p.189-202, 2004.

CAPARROZ, M. B. Ambiente, urbanização e agroindústria: a especificidade de Lucas do Rio Verde-MT. In: ENCONTRO NACIONAL DE ESTUDOS POPULACIONAIS, 17., 2010, Caxambu. Anais... Caxambu: UNESP, 2010. p. 1-20. Disponível em: $<\mathrm{ftp}$ //ftp.fao.org/docrep/fao /010/i0139e/i0139e03.pdf $>$. Acesso em: 03 fev 2018.

CHURCHMAN, G. J.; FOSTER, R. C.; D’ACQUI, L. P.; JANIK, L. J.; SKJEMSTAD, J. O.; MERRY, R. H.; WEISSMANN, D. A. Effect of land-use history on the potential for carbon sequestration in an Alfisol. Soil and Tillage Research, v. 109, n. 1, p. 23-35, 2010. DOI: https://doi.org/10.1016/j.still.2010.03.012

DAVIN, E. L.; NOBLET-DUCOUDRÉ, N. Climatic impact of global-scale deforestation: radiative versus nonradiative processes. Journal of Climate, v. 23, n. 1, p. 97-112, 2010. DOI: https://doi.org/10.1175/2009jcli3102.1

EMPRESA BRASILEIRA DE PESQUISA AGROCUÁRIA EMBRAPA. Árvore do conhecimento do milho: plantio direto. (2011). Disponível em: $<$ http://www.agencia.cnptia.embrapa.br/gestor/milho/arvo re/CONTAG01_72_59200523355.html>. Acesso em: 20 fev 2018.

ESPÍRITO-SANTO, F. D. B.; SHIMABUKURO, Y. E.; ARAGÃO, L. E. O. C.; MACHADO, E. L. M. Análise da composição florística e fitossociológica da floresta nacional do Tapajós com o apoio geográfico de imagens de satélites. Acta Amazônica, v. 35, n. 2, p. 155-173, 2005. DOI: https://doi.org/10.1590/s004459672005000200006

EXELIS VISUAL INFORMATION SOLUTIONS - ENVI User Manual. 2014.

GALFORD, G. L.; MELILLO, J. M.; KICKLIGHTER, D. W.; CRONIN, T. W.; CERRI, C. E.; MUSTARD, J. F.; CERRI, C. C. Greenhouse gas emissions from alternative futures of deforestation and agricultural management in the southern Amazon. Proceedings of the National Academy of Sciences, v. 107, n.46, p.19649-19654, 2010. DOI: https://doi.org/10.1073/pnas.1000780107

INSTITUTO BRASILEIRO DE GEOGRAFIA E ESTATÍSTICA - IBGE. Censo agropecuário 2006 Lucas do Rio Verde. Rio de Janeiro, 2006. Disponível em: $<$ https://cidades.ibge.gov.br/brasil/mt/lucas-do-rioverde/pesquisa/24/27745>. Acesso em: 06 fev 2018.

INSTITUTO BRASILEIRO DE GEOGRAFIA E ESTATÍSTICA - IBGE. Levantamento sistemático da produção agrícola. n. 09, p. 1-77. Rio de Janeiro, 2017. Disponível em: https://biblioteca.ibge.gov.br/visualizacao/periodicos/241 5/epag_2017_set.pdf >. Acesso em: 20 fev 2018. 
INSTITUTO BRASILEIRO DE GEOGRAFIA E ESTATÍSTICA - IBGE. Manual técnico de uso da terra. Manuais técnicos em geociências. 3. ed. n.7. Rio de Janeiro: IBGE, 2013. 170p. Disponível em: $<$ https://biblioteca.ibge.gov.br/visualizacao/livros/liv8161 5.pdf>. Acesso em: 16 fev 2018.

INSTITUTO MATO-GROSSENSE DE ECONOMIA AGROPECUÁRIA - IMEA. Relatórios de mercado soja safra 17/18. Disponível em: $<$ http://www.imea.com.br/imea-site/relatorios-mercado $>$. Acesso em: 01 jun 2018.

JUNIATI, E.; ARROFIQOH, E. N. Comparison of pixel-based and object-based classification using parameters and nonparameters approach for the pattern consistency of multi scale landcover. International Archives of the Photogrammetry, Remote Sensing \& Spatial Information Sciences, v. 42, 2017. DOI: https://doi.org/10.5194/isprsarchives-xlii-2-w7-765-2017

LANDIS, J. R; KOCH, G. G. The measurement of observer agreement for categorical data. Biometrics, v. 33, n. 1, p. 159-174, 1977. DOI: https://doi.org/10.2307/2529310

MELLO, I. A. Y.; SALAS ALVES, D.; ALBUQUERQUE LINHARES, C.; BUENO DE LIMA, F. Avaliação de técnicas de classificação digital de imagens Landsat em diferentes padrões de cobertura da terra em Rondônia. Revista Árvore, v. 36, n. 3, 2012. DOI: https://dx.doi.org/10.1590/s0100-67622012000300016

MINISTRO DE ESTADO DO MEIO AMBIENTE - MMA. Portaria $n^{\circ}-361$, de 8 de setembro de 2017. Tabela de municípios prioritários para ações de prevenção, monitoramento e controle do desmatamento. Diário Oficial [da] República Federativa de Brasil, Brasília, p. 69-70, 13 set. 2017. Seção 1. Disponível em: $<$ http://pesquisa.in.gov.br/imprensa/jsp/visualiza/index.js p? jornal $=1 \&$ pagina $=69 \&$ data $=13 / 09 / 2017>$. Acesso em: 20 fev 2018.

NEPSTAD D. C.; STICKLER C. M.; FILHO B. S.; MERRY F. Interactions among Amazon land use, forests and climate: prospects for a near-term forest tipping point. Philosophical Transactions of the Royal Society of London B: Biological Sciences, v. 363, n. 1498, p. 17371746, 2008. DOI: https://doi.org/10.1098/rstb.2007.0036

NOVO, M. L. E. Sensoriamento Remoto: Princípios e Aplicações. 4. ed. São Paulo: Edgard Blücher Ltda, 2010, $388 \mathrm{p}$.

PRADHAN, B. B.; SHRESTHA, R. M.; HOA, N. T.; MATSUOKA, Y. Carbon prices and greenhouse gases abatement from agriculture, forestry and land use in Nepal. Global Environmental Change, v. 43, p. 26-36, 2017. DOI: https://doi.org/10.1016/j.gloenvcha.2017.01.005

RAHMAN, A. F.; GAMON, J. A.; FUENTES, D. A.; ROBERTS, D. A.; PRENTISS, D. Modeling spatially distributed ecosystem flux of boreal forest using hyperspectral indices from AVIRIS imagery. Journal of Geophysical Research, v. 106, n. 24, p. 33.579-33.591, 2001. DOI: https://doi.org/10.1029/2001JD900157

RAHMAN, A.F.; GAMON, J.A.; FUENTES, D.A.; ROBERTS, D.; PRENTISS, D.; QIU, H. Modeling $\mathrm{CO}_{2}$ flux of boreal forests using narrow-band indices from AVIRIS imagery. In: AVIRIS WORKSHOP. Anais... Pasadena: JPL/NASA, 2000. Disponível em: $<$ https://aviris.jpl.nasa.gov/proceedings/workshops/00_do cs/Rahman_web.pdf $>$. Acesso em: 02 fev 2018

RIZZI, R.; RUDORFF, B. F. T. Estimativa da área de soja no Rio Grande do Sul por meio de imagens Landsat. Revista Brasileira de Cartografia, v.3, n.57, p.30-35, 2005. DOI: https://doi.org/10.1590/s0103-84782006000100005

SÁ, J. C. M, SÉGUY, L., TIVET, F., LAL, R., BOUZINAC, S., BORSZOWSKEI, P. R., ... \& ROSA, J. CARBON depletion by plowing and its restoration by no-till cropping systems in oxisols of subtropical and tropical agroecoregions in Brazil. Land Degrad. Dev, v. 26, n. 6, p.531-543, 2015. DOI: https://doi.org/10.1002/ldr.2218

SILVA, S. C. P.; BAPTISTA, G. M. M. Análises espectrais da vegetação com dados hyperion e sua relação com a concentração e o fluxo de $\mathrm{CO} 2$ em diferentes ambientes na Amazônia brasileira. Boletim de Ciências Geodésicas, v. 21, n. 2, p. 354-370, 2015. DOI: http://dx.doi.org/10.1590/S1982-21702015000200020

SISTEMA DE ESTIMATIVAS DE EMISSÕES E REMOÇÕES DE GASES DE EFEITO ESTUFA -SEEG - BRASIL (2018). Emissões totais. Disponível em $<$ http://plataforma.seeg.eco.br/total_emission>. Acesso em: 06 de fev 2018.

SOUZA, A. P.; MOTA, L. L.; ZAMADEI, T.; MARTIN, C. C.; ALMEIDA, F. T.; PAULINO, J. Classificação climática e balanço hídrico climatológico no estado de Mato Grosso. Nativa, v. 1, n. 1, p. 34-43, 2013. DOI: https://doi.org/10.14583/2318-7670.v01n01a07

TEOBALDO, D.; BAPTISTA, G. M. Quantificação da severidade das queimadas e da perda de sequestro florestal de carbono em unidades de conservação do Distrito Federal. Revista Brasileira de Geografia Física, v. 9, n. 1, p. 250-264, 2016. DOI: https://doi.org/10.26848/rbgf.v9i1.1199

WANDERLEY, L. J. Repensando a noção de fronteira no contexto de reestruturação espacial da Amazônia no século XXI. Terra Livre, v. 1, n. 46, p. 13-48, 2018. Disponível em: https://rbg.ibge.gov.br/index.php/rbg/article/view/110 >. Acesso em: 20 de jun de 2018.

YUE, Q., XU, X., HILLIER, J., CHENG, K., \& PAN, G. Mitigating greenhouse gas emissions in agriculture: From farm production to food consumption. Journal of Cleaner Production, v. 149, p. 1011-1019, 2017. DOI: https://doi.org/10.1016/j.jclepro.2017.02.172 